\title{
Confrontation of Filter Banks Conception Procedures for Attributes Extraction of the Airborne SAR Image Textures
}

\author{
Marrakchi Charfi Olfa, Mbainaibeye Jérôme
}

\begin{abstract}
This study consists of evaluating the performance and the accuracy of three filter bank design methodologies for the identification of the complex textural primitives of a SAR satellite image (4.6 meter resolution). The development of these filters is made in order to provide an accurate analysis of the available SAR images. For this purposes, we have used the following orthogonal spatial transformations: Karhunen Loeve Transform (KLT), Gabor Filters (GF) and Generalized Gaussian Modeling (GGM) by Wavelet Transform (WT), which allow the reduction of the correlation between data. The filter banks are represented by a combination of the optimal filters, selected for each texture according to the used transform. This paper shows the difficulty to choice the adequate design of the filter banks that allows an optimum identification of the textures of SAR images and classification of these images based on their filtered attributes. The results showed that the conventional filters used in the WT are not accurate for the heterogeneous textural identification, while high identification rate exceeding 99\% can be obtained with an adequate combination of the filters. The best filter bank is the one that allows the identification of all textures in spite of their dimension.
\end{abstract}

Index Terms - Filter Banks, Image Textures, Texture Extraction, Filter Combination

\section{INTRODUCTION}

The identification of textures of satellite images has become a common practice used in many fields such as agriculture and forestry, and several works have been published on this topic. However, this task becomes harder when the textures are heterogeneous and irregular, requiring the use of appropriate methodologies and sometimes the adjustment of certain data. The Gabor filters, which are known for their robustness, have been widely used to a broad range of image processing tasks.

The Gabor filters are very popular due to their conjoint space-frequency resolution which is optimal [8]. However, the design of the specific filters must be realized with high precision if the detection of the textural forms is required. Recently, Zand and al. [16] have used the Gabor and Curvelet approaches for the identification of the different textures of satellite images and modeling. However, as the Gabor and Curvelet transforms are designed for the regular shapes, only, a fitting method was applied to encode the information of the sub-bands in the polynomial coefficients, giving a texture feature vector with a maximum power of discrimination. For all these reasons and others, other approaches are proposed. Indeed, it was shown that it is possible to use the GGM of the

Marrakchi Olfa Charfi, National Institute of Applied Science and Technologies, Member of GreenTeam-INAT LR17AGR01 Laboratory, Tunis, Tunisia

Mbainaibeye Jérôme, University of Doba, Doba, Chad local variances distributions of the energies of the sub-images issued from the WT to have a good classification of the different textures present within the remotely sensing images, amongst the SAR image [15]. With this method, it is also possible to develop the textures filter banks as we did in the following sections.

The orthogonal transformations such as: KLT [3], WT [4], frames representation for textures [5], GF [9], were found to be more appropriate for classifying complex textures, allowing an optimal segmentation of the remote sensing images. It was observed that some descriptors such as the textural signatures of the SAR image textures, which are determined in the spatial representations using the KLT, WT and GF tools, are more efficient in characterizing such image textures [1]. Indeed, the KLT contributes to increase the capacity of the discrimination procedure of the objects inside the images. The information obtained from the new KLT axis constitutes the statistic information, which allows an easy identification of the studied texture. This image may be decomposed in the new orthogonal basis by filtering. The data obtained in the new basis are uncorrelated [2][3].

The WT consists in analyzing a signal by using a localized centered function which may be translated and dilated [4], providing uncorrelated data [5] and allowing a space-frequency analysis identification of the global and the local textural informations. The WT is based on two conjugate filters: low pass filter and high pass filter, which are, also called quadrature mirror filters (QMF)[6]. With the WT tool, it is possible to have a decomposition of an image into sub bands called low pass sub band and high pass sub bands, which contains the details of the image [7]. Thus, the complexity of the data, their dimension and their inter relationships strongly influence the credibility of the results that can be obtained and define the degree of difficulty in analyzing the heterogeneous textures. In such analysis, it is important to localize the information at both, the spatial domain to characterize the orientations of the primitives and at the frequential domain to obtain the grey scales variation that can be used as descriptors; in this context, the best conjoint localization (spatial and frequential domains) may be obtained by the Gabor filtering [9][10], with the cosine filter, which has a certain direction modulated by a Gaussian window [11][12].

In this paper, we are interested in the development of texture filter banks which are used to analyze the textures primitives and their attribute images. The remaining of this paper is organized as follows: In the section 2, we present the methods and materials used; the section 3 presents the results obtained; the section 4 presents the discussions of these 
results; finally, the conclusion of this work is presented in the section 5 .

\section{METHODS AND MATERIAL}

\section{A. Filter bank design based on transform}

\section{A.1 The KLT [1]}

The KLT image based transform is operated locally by considering a narrow neighboring, which is represented by a random vector $X$ (its values are taken in the observation window where the dimension is $k_{b} x k_{b}$ with $k_{b} x k_{b}=N$. The different realizations of $X$ are obtained by moving the observation window through the image. For each value of $X$ and for a given spatial position, is attributed its image $Y$ by a linear transform represented by equation (1). The vector $Y$ represents the image of the vector $X$ in an orthonormal basis $\phi$ given by equation (2) and contains $\mathrm{N}$ eigenvector of the $\mathrm{X}$ covariance matrix.

$$
Y=\phi^{T} X
$$

Where:

$$
Y=\left(\begin{array}{c}
y_{1} \\
y_{2} \\
\vdots \\
\vdots \\
y_{N}
\end{array}\right)
$$

$$
X=\left(\begin{array}{c}
x_{1} \\
x_{2} \\
\vdots \\
\vdots \\
x_{N}
\end{array}\right)
$$

The orthonormal basis is given by equation 2 :

$$
\phi=\left[\begin{array}{ccc}
\phi_{1,1} & \cdots & \phi_{1, N} \\
\vdots & \cdots & \vdots \\
\phi_{N_{J} 1} & \cdots & \phi_{N_{N}, N}
\end{array}\right]
$$

According to [2], it is possible to establish a correspondence between the linear transform of a vector $X$ by an eigenvector $\phi_{n}$ and the dimensional convolutional product of the observation of the window $X(i, j)$ and a Finite Impulse Response filter (FIR) $\phi_{n}(i, j)$ represented by equation ( 3 ). This filter is constituted of the eigenvector coefficients reordered according to their positions in the observation window $X(i, j)$.

$$
Y(i, j)=X(i, j) * \phi_{n}(i, j)
$$

Where $\phi_{n}\left(i_{i} j\right)$ is given by equation (4).

$$
\phi_{n}\left(i_{x} j\right)=\left[\begin{array}{ccc}
\Phi_{1, n} & \cdots & \vdots \\
\vdots & \cdots & \vdots \\
- & \cdots & \phi_{N_{n} n}
\end{array}\right]
$$

The image described by the variable $Y(i, j)$ is called image attribute or filtered channel. A filter bank is composed of the

FIR filters $\phi_{n}(i, j)$ with $1 \leq n \leq N_{\text {. }}$

\section{A.2 The GGM [1][13]}

To local variance distributions of each detail subband energies of the image obtained by the WT at several scales

may be associated to a scale function model $\Phi(x)$. The first derivative of this function represents the wavelet function $\Psi(x)$ [4]. From the Gaussian model values of the function $\Phi(x)$ [7] and its first derivative $\Psi(x)$, the impulse responses of the filters $h(k)$ and $g(k)$ are calculated by the resolution of an equation system depending on $\Phi(x)$ and $\Psi(x)$ expressed by equation (5).

$$
\left\{\begin{array}{l}
\Phi(x)=\sqrt{2} \Sigma_{k} h(k) \Phi(2 x-k) \\
\Psi(x)=\sqrt{2} \Sigma_{k} g(k) \Phi(2 x-k)
\end{array}\right.
$$

All couples of filters $(\mathrm{h}, \mathrm{g})$ designed in several scales represent the filter banks that we call Generalized Gaussian Model Filter Bank (GGM-FB). Figure 1 represents the scheme of the wavelet decomposition in one scale.

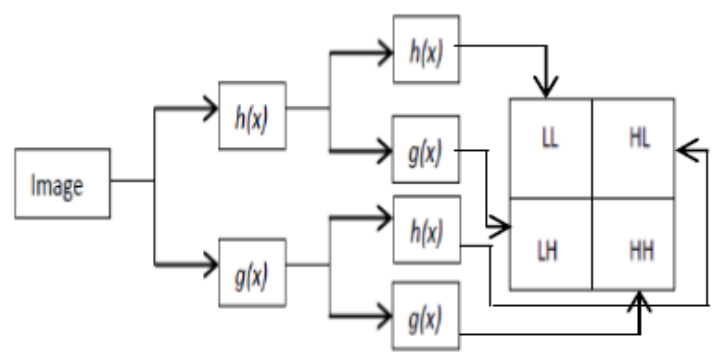

Fig.1. One scale image wavelet decomposition.

\section{A.3 The Gabor filters [13]}

The approach developed in this work, is based on a multichannel filtering of the image in a set of images containing the spatial characteristics at different scales. Additionally, the original image is decomposed in different planes corresponding to different frequential channels. Each of these images contains the textural characteristics appearing in a narrow spatial frequency band and orientation. This approach has the advantage to exploit the spatial interactions between pixels of neighboring at different scales. After filtering, we have selected the filters which are able to offer the capacity for efficiently detecting of the texture motives. The best simultaneous localization in spatial and frequential domain may be operated by the Gabor filtering (see equation 6) applying a cosine filter with a certain direction modulated by a Gaussian window [11][12]. The radar image used in this work has only the real discrete values. The real form of the Gabor filter is defined by equation (7), where $\sigma_{x}$ and $\sigma_{y}$ are the Gaussian standard deviations, $f_{x_{0}}$ and $f_{y_{0}}$ are the central frequencies, respectively in the $\mathrm{x}$ and $\mathrm{y}$ axis. To obtain different orientations, a rotation of the coordinate axis is made by using equation (8).

$$
\begin{aligned}
& g_{b}(x, y)=\frac{1}{2 \pi \sigma_{x} \sigma_{y}} \exp \left(-\frac{x^{2}}{2 \sigma_{x}^{2}}-\frac{y^{2}}{2 \sigma_{y}^{2}}\right) \exp \left(j 2 \pi \left(f_{x_{0}} x+\right.\right. \\
& \left.\left.f_{y_{0}} y\right)\right)
\end{aligned}
$$

$$
g_{b}\left(x_{i}, y_{i}\right)=\frac{1}{2 \pi \sigma_{x} \sigma_{y}} \exp \left(-\frac{x_{i}^{2}}{2 \sigma_{x}^{2}}-\frac{y_{i}^{2}}{2 \sigma_{y}^{2}}\right) \cdot \cos \left(2 \pi f _ { 0 } \left(x_{i}+\right.\right.
$$$$
\text { Y)) }
$$

Where

$\left(\begin{array}{l}x_{i} \\ y_{i}\end{array}\right)$ is given by equation (8):

$$
\left(\begin{array}{l}
x_{i} \\
y_{i}
\end{array}\right)=\left(\begin{array}{cc}
\cos \theta_{i} & \sin \theta_{i} \\
-\sin \theta_{i} & \cos \theta_{i}
\end{array}\right)\left(\begin{array}{l}
x \\
y
\end{array}\right)
$$


With

$$
\theta_{i}\left\{\begin{array}{lll}
=0 & \text { for } & i=0 \\
\neq 0 & \text { for } & i \neq 0
\end{array}\right.
$$

The GF has four parameters: the filter orientation, the central frequency $f_{0}$ to be extracted, the standard deviations $\sigma_{x}$ and $\sigma_{y}$. For each texture, the GF are determined and permits its identification depending of the following variables: $\theta_{i}, \sigma_{\mathrm{x}}\left(\sigma_{\mathrm{x}}=\sigma_{\mathrm{y}}\right)$ and $f_{0}$. Also, for each texture, a judicious choice of an optimal filter is realized among this set of filters according to the imposed criteria to its textural identification. The GF Filter Bank (GF-FB) is in this case composed of the combination of the optimal filters associated to each of the textures.

\section{B. Texture descriptor}

\section{B.1 Signature used for the KLT method [1]}

The signature vector $C_{K L T}$ attributed to each texture is formed by the energy eigenvalues of the texture; this vector is given by equation (9), where $\lambda_{\hat{I}}$ is the $i^{\text {th }}$ energy eigenvalue extracted from the correlation matrix of a given texture and $N$ represents the number of the energy eigenvalues.

$$
C_{K L T}=\left(\lambda_{1}, \lambda_{2, \ldots,} \lambda_{N}\right)(9)
$$

The number of the filter banks may be limited to a value lower than $\mathrm{N}$ according to the energy ratio $E R$ (see equation $10) . E R$ is determined from the eigenvalues associated to the eigenvectors of the covariance matrix of the observation

vector $X$, with $\lambda_{1}>\cdots>\lambda_{N}$ and $p \leq N$

$$
E R(\%)=\frac{\sum_{i=1}^{D} \lambda_{\mathrm{i}}}{\sum_{\mathrm{i}=1}^{\mathbb{N}} \lambda_{\mathrm{i}}}
$$

\section{B.2 Signature used for GF method}

The signature vector, $C_{G F}$, attributed to each texture is composed of two components determined on the filtered images by all the filters of the optimal filter bank. These components are expressed in terms of energy E and L1 norm $V$ given respectively by equations (12) and (13). This vector is given by equation (11), where $E_{i j}$ defines the energy of the GF coefficients of texture $T_{i}$ after filtering by the GF of frequency $f_{j}, V_{i j}$ is the $L 1$ norm obtained from the output of GF of frequency $f_{\mathrm{j}}$. If a bank of four filters is used, the characteristic vector is written as indicated in equation (14), where $E_{i l}$ (respectively $V_{i I}$ ) represents the energy (respectively norm L1) of the GF coefficients for the frequency $f_{i}$ of the texture $i$.

$$
\begin{aligned}
& C_{F G}=\left(E_{i j}, V_{i j}\right) \\
& E_{i j}=\frac{\sum_{i=1}^{P} \sum_{j=1}^{Q}\left[\left(\mathrm{i}_{i j} j\right)^{2}\right.}{P Q} \\
& V_{\mathrm{i} j}=\frac{\left.\sum_{\mathrm{i}=1}^{P} \Sigma_{j=1}^{Q} \mid r(\mathrm{i}, j)\right]}{P Q} \\
& C_{\mathrm{i}}=\left(E_{\mathrm{i} 1}, E_{\mathrm{i} 2}, E_{\mathrm{i} a}, E_{\mathrm{i} 4}, V_{\mathrm{i} 1}, V_{\mathrm{i} 2}, V_{\mathrm{i} a}, V_{\mathrm{i} 4}\right)
\end{aligned}
$$

\section{B.3 Signature used for the GGM method}

The signatures in terms of energy (E) norm L1 (V) are determined in the different detail subbands HL, LH and HH for the different scales of image wavelet decomposition. The signature vector $V_{W T-G G M}$ is determined by equation 15 , where $E_{i}$ is the energy signature and $V_{i}$ is the L1 norm signature; $E_{i}$ and $V_{i}$ are determined in the $i^{\text {th }}$ detail subband at a given scale.

$$
V_{W T-G G M}=\left(E_{H L}, E_{L H}, E_{H H}, V_{H L}, V_{L H}, V_{H H}\right)
$$

\section{Classification method}

The image classification based on minimum distance is realized according to the minimization of the mean square error (MSE) between the local statistics calculated in the scanning windows of $k_{b} x k_{b}$ size on the filtered images and the textural signatures determined in the localized textural zones on the filtered images. The minimization of the $M S E$ is given by equation (16), with $c_{j}$ the $j^{\text {th }}$ component of the local statistic

vector, $C_{j}^{T_{\mathrm{i}}}$ the jth component of the characteristic vector of

the texture $T_{i}$ and $n$ represents the number of the vector components which is a variable parameter depending on the selected transform method.

$$
\min _{T_{i}}(E Q M)=\sum_{j=1}^{n}\left(c_{j}-C_{j}^{T_{i}^{i}}\right)^{2}
$$

The image resulting from the minimum distance classification is an image of labels. The label represents the index $i$ of the texture $T_{i}$ having a minimal MSE.

The local statistics which are calculated in the scanning windows $k_{b} x k_{b}$ are different from one transform to another:

- For KLT, the local statistics are only represented by the average energy calculated according to equation (12) and determined in the observation windows on all the image attributes. In this case, the local statistic vectors $c$ and the characteristics of the textures $C$ have a number of components equal to the number of image attributes. The image is filtered by the filters of each filter bank (relative to a texture). All the image attributes by filter bank represent a list of images $L_{i}$ associated to a given texture $T_{i}$. In the classification phase, the $M S E$ is determined for each $L_{i}$ and for each position of the observation vector $X$. For a given position of $X$, the associated class label is those of the texture for which $M S E_{i}$ is the smallest for $i=1$ to 4 . This classification procedure is based on the method presented in [3];

- For WT-GGM, the local statistics are defined in terms of energy (see equation 12), and L1 norm determined in the observation windows for all the images subbands for all the scales. In this case, the vectors (local statistics $c$ and the textural characteristics $C$ ) have a number of components twice the number of the image detail subbands for $K$ wavelet decomposition levels;

- For GF, the local statistics are defined in terms of energy (see equation 12), and L1 norm determined in the observation windows for all the filtered images by the filter banks. In this case, the vectors (local statistics $c$ and the textural characteristics $C$ ) have a number of components twice the number of the filtered images.

\section{Evaluation criteria}

The filter banks are evaluated according the classification result by minimum distance of the SAR image textures in 
terms of identification rate given by equation (17), with $c$ the number of classes, $I_{s}$ the label of pixel $s$ and $p\left(I_{s}=I / T_{s}=T\right)$ is the conditional probability that the pixel $s$ is classified in class $I$ as it is in the texture $T$.

$$
\operatorname{lr}(T)=\sum_{i=1}^{c}\left(p\left(I_{g}=i / T_{g}=T\right) / p\left(T_{g}=T / I_{g}=i\right)\right) \times
$$
100

The validation of the filter banks is performed according to the chart shown in figure 2 .

The image is filtered by all the filters according to the applied transform method. From the filtered images and from the ground truth data location on these filtered images, the textural features are determined for each texture used for the airborne SAR image classification.

The classification criterion used is the Mean Squared Error (MSE) between the local descriptor, calculated from the scanning windows $\left(k_{b} x k_{b}\right)$ on filtered images (or attributes) and their textural signatures.

The signatures are different from one method to another (KLT, GGM and GF). For some methods, a restriction on the number of filters in a filter bank is realized for the reduction of the computation amount in the filtering phase but keeping the maximum information of the image.

The evaluation of the information is realized in this case by the computation of the energy ratio in percent $(E R)$ expressed by equation (10).

The selected filter banks are those which allow obtaining the best classification results in terms of identification rate according to equation (17) in the case of GGM and GF transforms. However, for KLT, the optimal filter bank is selected after the verification of the work presented in [3].

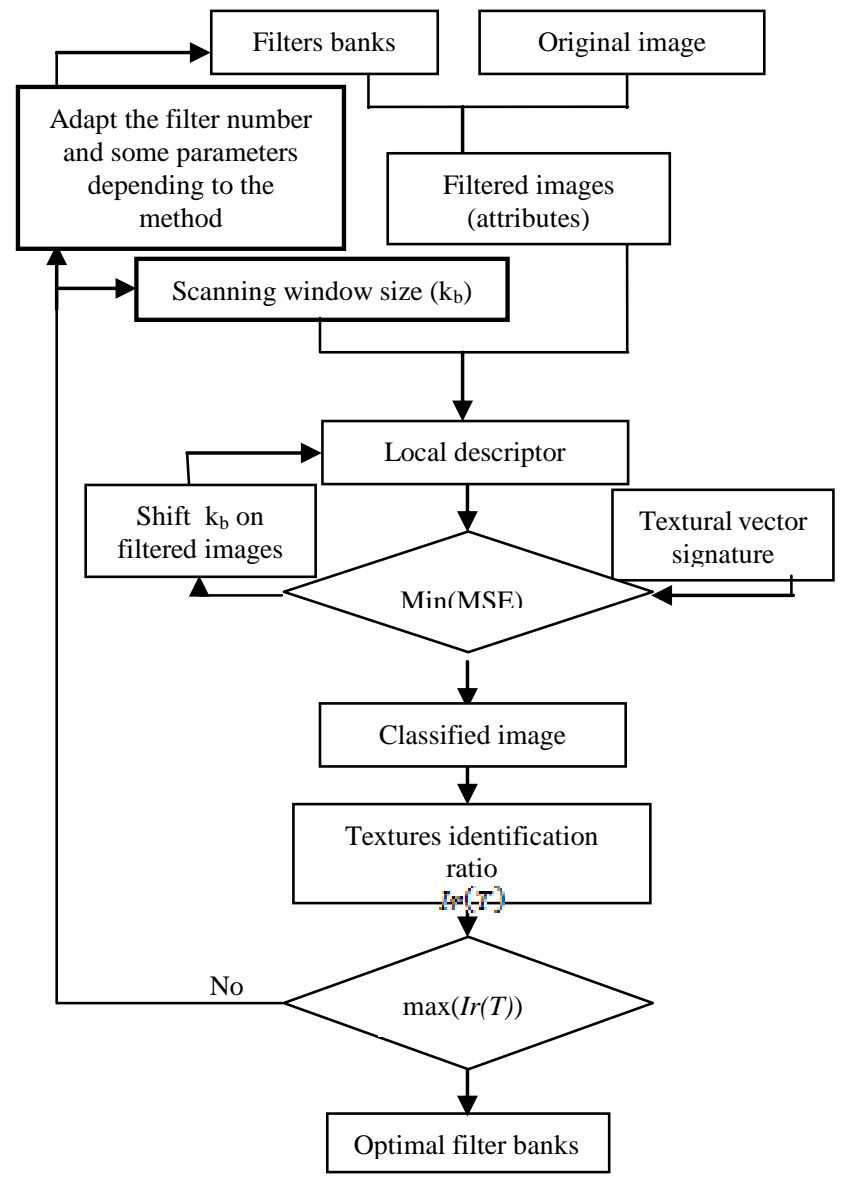

Fig. 2. Filter bank validation flow chart

\section{E. Datasets}

The textures used in this work are extracted from an image obtained by an airborne SAR in $\mathrm{C}$ band, in horizontal polarization and the resolution is 4.6 meters (Figure 4). This image covers a semi-arid region located in southern Tunisia. According to a ground truth campaign, soils in this region are essentially covered with scattered vegetation (V), olive trees (O.V), bare soils (BG.V) and urban areas (B.V). The thematic studies done on the zone have enabled us to identify the different textures on the airborne SAR image. This image has been the subject of several years of study since 1994. All texture classification operations have been performed on this image using several methods [13][14]. Moreover, a comparative study of classification results with those of another radar image, acquired with RADARSAT satellite with lower resolution (12.5 meters), was performed before seeing the influence of the spatial resolution and its impact on the classification results [1]. We have deduced that the primitives in textures of the airborne SAR image are easily identified compared to those of the RADARSAT image. Hence, the interest is shown in these textures for developing and studying the performance of filter banks.

The SAR image textures are irregular and consist of a common textural element which explains the strong correlation between the textures. For example, the textures O.V and BG.V have the "bare ground" as a common structural element; this is observed in figure 3 .

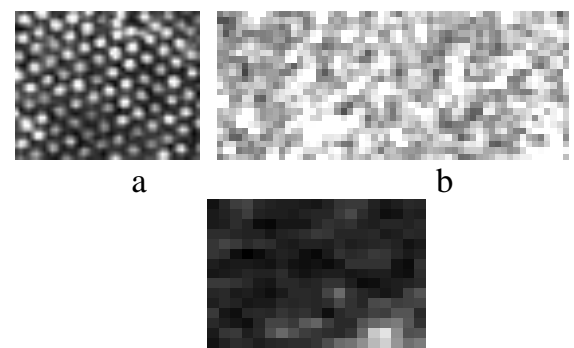

Fig. 3. Example of SAR image windows. (a) Olives on the ground covered with vegetation $O . V(37 \times 47$ pixels), (b) Built on ground covered with vegetation B.V (23 $x 57$ pixels), (c) bare ground with the presence of vegetation BG.V( $15 \times 19$ pixels), (d) vegetation $V(21 \times 37$ pixels $)$.

The SAR textures are complex and are characterized by information redundancy. To reduce this textural correlation, the KLT [1], the WT and the GF [13] are used to represent these data in other spaces by conserving the global information of these textures [1][14][15].

\section{RESULTS}

\section{A. Textural filter banks elaboration by the KLT}

For each texture, the filter banks and the associated textural signature vector (equation 9) are determined. The SAR image is filtered by the different elaborated filter banks and then, it is classified by the minimum distance method. Three parameters are adjusted for the optimization of the results:

- The scanning window width $k_{b} x k_{b}$, used in the image classification phase;

- The window of dimension $l \times l$ represented by a given textural texton used in the verification of the [3];

- The value attributed to energy ration $E R$. 
The best SAR image classification results are obtained for $k_{b}=9,1=7$ and $E R=90 \%$

The best average identification rate is $80.2 \%$ as shown in Tab.2.

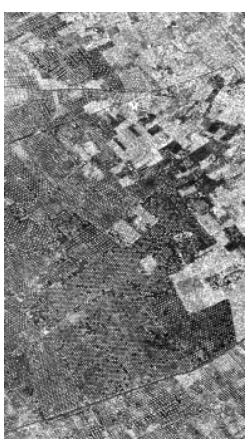

Fig. 4. Airborne SAR image in $C$ band, in horizontal polarization with resolution of 4.6 meters

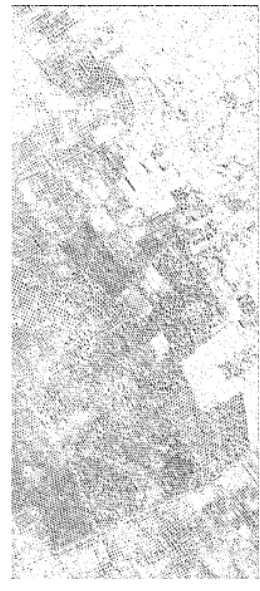

a

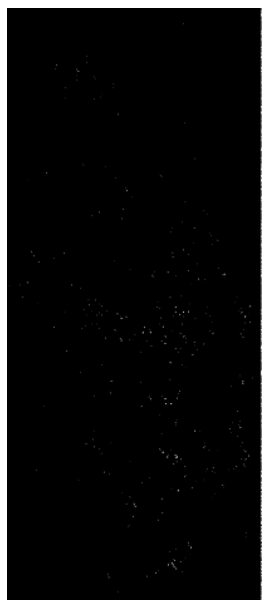

$c$

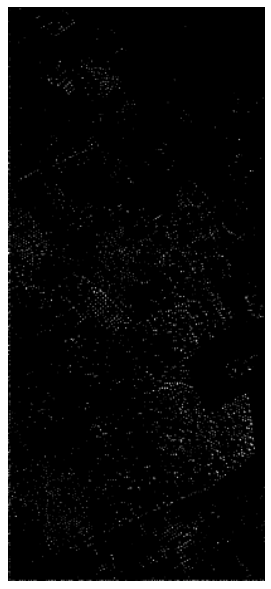

b

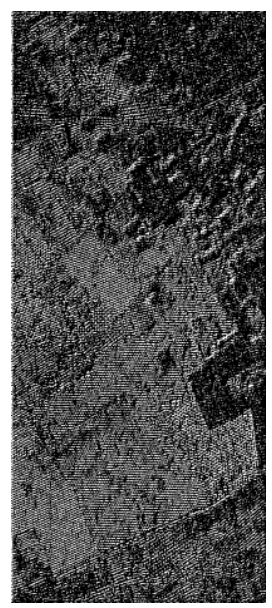

$d$
Fig.5. The first image attribute associated to the first more important energies for each texture (KLT method):

(a) for O.V texture, (b) for B.V texture, (c) for BG.V texture and $(d)$ for $V$ texture

Figure 5 illustrates the results of the KLT applied on SAR images and shows the first image attribute for each texture.

This study shows that the number of filters varies from one texture to another (see Tab.1) and the computation time may be considered as a constraining parameter because the number of filtering operations is high. Only the eigenvalues associated to the filters (or eigenvectors) are illustrated in figure 6 and they represent the textural signatures.

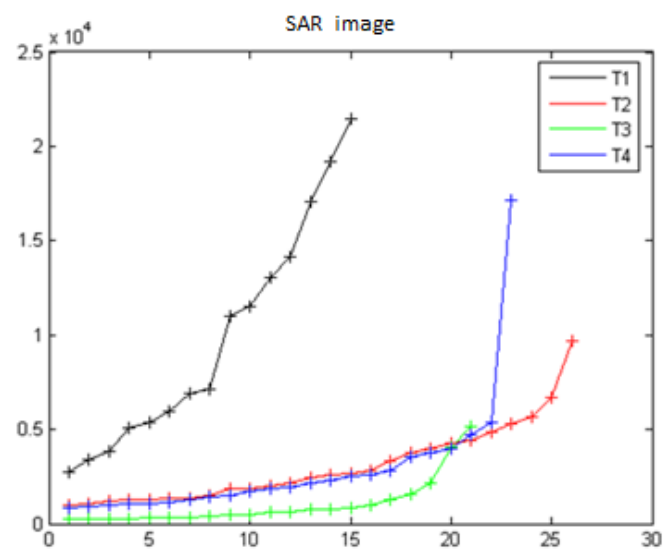

Fig.6. Variation of signature vector coefficients of SAR image texture for $l=7$ and $E R=90 \%$. KLT method

Table.1. Number of filters by texture for the SAR image transformed by KLT. $E R=90 \%$ and $l=7$

\begin{tabular}{ccccc}
\hline Textures & O.V & B.V & BG.V & V \\
\hline Number of filters & 15 & 26 & 21 & 23 \\
\hline
\end{tabular}

Table.2 Identification ratio of SAR image textures in \%.

\begin{tabular}{ccccc}
\hline O.V & B.V & BG.V & V & $\begin{array}{c}\text { Average } \\
\text { Identification ratio (\%) }\end{array}$ \\
\hline 58.80 & 92.90 & 86.40 & 82.70 & 80.20 \\
\hline
\end{tabular}

\section{B. Textural filter banks elaboration by the GGM}

The best classification results by minimum distance using GGM of the distributions of local variances in the detail subbands of SAR image was achieved for a size of scanning window $k_{b}=11$ and for two scales [15].

The scale function and wavelet function have been modeled on each scale of WT. By using these parameters and these models, and by solving the system of equations, the filters $h$ and $g$ were determined on each scale of WT for each of the textures in the image. Thus, for each texture are associated two filter banks: 1 bank by 2 scales of WT. Each filter bank is composed of three couples $(h, g)$ extracted from the detail subbands: HL, LH and HH. Subsequently, the filter banks elaborated are used to realize the WT of the original image on two scales (Figures 7 and 8).

The principle of the WT has been applied on the SAR image by using the filter banks $h$ and $g$ realized (see Table 3 ). Thus, to get the horizontal detail subband image (HL), we applied the high pass filter $g_{H L}$, on the rows and then the low pass filter, $h_{H L}$, on the columns of the original image (or the approximation subband if it concerns the second scale of WT). To get the vertical detail subband image (LH), we applied the low pass filter, $h_{D V}$, on the rows and then the high-pass filter, $g_{D V}$, on the columns of the original image (or the approximation subband if it concerns the second scale of WT) and finally, to get the diagonal detail subband image $(\mathrm{HH})$, we applied the high pass filter, $g_{D D}$, on the rows. This procedure is repeated according to the scale number in WT decomposition. 
The best identification rates of the SAR image textures were obtained using the signatures of textures based on the detail subbands after application of WT using the filter banks of the texture B.V on one scale and for a scanning window size which is $k_{b}=11$ (see Fig.9). Thus, the average rate of good classification of the four textures of SAR image reached $91.31 \%$ (see Tab.4). Note that the identification rate obtained for BG.V texture is $100 \%$.
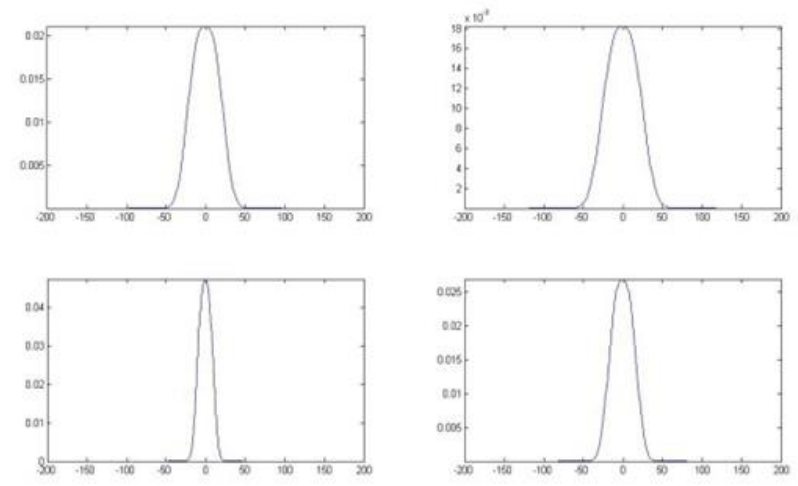

Fig.7. Example of scale functions obtained from the GGM of $H H$ detail subbands of SAR image textures. (a) Texture $O . V$, subband HH, 1 scale. (b) Texture B.V, subband $H H$,

1 scale. (c) Texture BG.V, subband HH, 1 scale. (d) Texture $V$, subband $H H, 1$ scale.
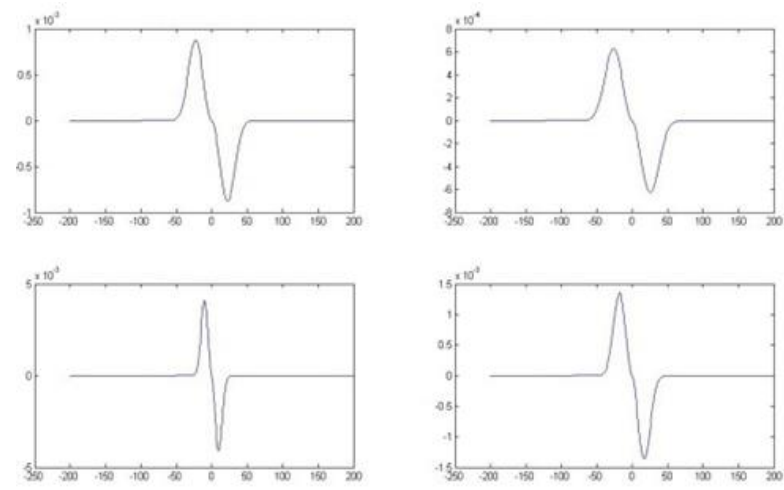

Fig.8. Example of wavelet functions obtained from the GGM of HH detail subbands of SAR image textures. (a) Texture O.V, subband HH, 2 scales. (b) Texture B.V, subband HH, 2 scales. (c) Texture BG.V, subband HH, 2 scales. (d) Texture $V$, subband $H H, 2$ scales.

Table.3. Filter banks coefficients for the texture B.V of SAR image (one scale). (a) Filter $h$, (b) filter $g$

\begin{tabular}{cccc}
\hline $\begin{array}{c}\text { Coefficients } \\
\text { of filter } h\end{array}$ & DH & DV & DD \\
\hline $\mathrm{h}(1)$ & -0.5234 & -0.5086 & -0.5883 \\
$\mathrm{~h}(2)$ & 0.5593 & 0.5633 & 0.6716 \\
$\mathrm{~h}(3)$ & 0.6207 & 0.5766 & 0.4871 \\
$\mathrm{~h}(4)$ & 0.6482 & 0.6648 & 0.8396 \\
$\mathrm{~h}(5)$ & -0.5993 & -0.5918 & -0.7125 \\
\hline & & & \\
\hline Coefficients & $\mathrm{DH}$ & $\mathrm{DV}$ & $\mathrm{DD}$ \\
of filter g & & & 0.0098 \\
$\mathrm{~g}(1)$ & 0.0098 & 0.0094 & -0.0124 \\
$\mathrm{~g}(2)$ & -0.0091 & -0.0100 & 0.0150 \\
$\mathrm{~g}(3)$ & 0.0144 & 0.0145 & 0.0068 \\
$\mathrm{~g}(4)$ & 0.0003 & 0.0017 & -0.0191 \\
$\mathrm{~g}(5)$ & -0.0154 & -0.0156 & \\
\hline
\end{tabular}

Tab.4. Identification average rate of the SAR image texture for BF-MGG.

$k_{b}=11, \mathrm{~B} . \mathrm{V}$ texture filter bank for one scale

\begin{tabular}{ccccc} 
O.V & B.V & BG.V & V & Average rate (\%) \\
\hline 88.10 & 82.50 & 100 & 94.70 & 91.30
\end{tabular}

\section{Textural filter banks elaboration by FG}

The elaboration of Gabor filter banks requires two steps: firstly, we must determine, for each texture, different filters for its identification and furthermore for the enhancement of its textural patterns without alteration of their visual structure (see Tab.5). Then, several combinations of filters are realized: we choose a filter by texture, and then the selected filters are grouped to make a combination of filters. For each texture is attributed a textural signature $(E$, standard $L 1)$, determined on the image filtered through the optimal filter associated to this texture. The classification by minimum distance will indicate us what among the combinations of textural filters will achieve the best texture identification rate (see Tab.6). This method is more complex and requires a big computation time. It also requires a visual validation, (see Tab.6), so its realization cannot be automatic.

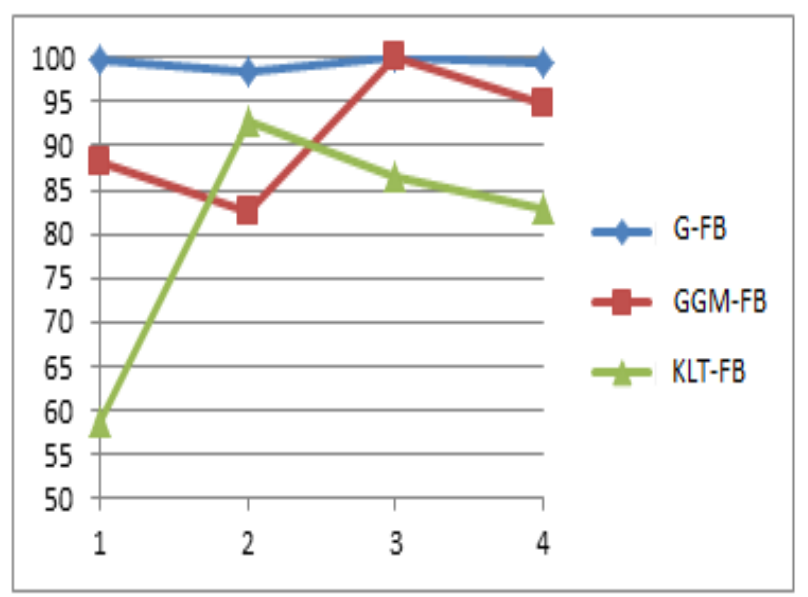

Fig. 9. Variation of the texture identification rates of $S A R$ textures by the differents filter banks: $G-F B, G G M-F B$ and KLT-BF

Table.5. The Gabor filter parameters chosen for each texture

\begin{tabular}{|l|l|}
\hline Textures & $\mathrm{f}_{0} \sigma, \theta$ values \\
\hline$O . V$ & $\begin{array}{l}\mathrm{f}_{0}=\{0.01,0.03,0.09,0.11,0.2,0.3,0.4,0.5\} \\
\sigma=0.5, \theta=0\end{array}$ \\
\hline$B . V$ & $\begin{array}{l}\mathrm{f}_{0}=\{0.01,0.09,0.2,0.3,0.4,0.5\} \\
\sigma=0.5, \theta=0\end{array}$ \\
\hline$B G . V$ & $\begin{array}{l}\mathrm{f}_{0}=\{0.01,0.09,0.11,0.2,0.3,0.5\} \\
\sigma=0.5, \theta=0\end{array}$ \\
\hline$V$ & $\begin{array}{l}\mathrm{f}_{0}=\{0.01,0.09,0.2,0.3,0.4,0.5\} \\
\sigma=0.5, \theta=0\end{array}$ \\
\hline
\end{tabular}


Table.6. Optimal textural identification rate of SAR image Gabor filter bank.

frequencies $=\left[0.09,0.09,0.01,0.09, \theta=0^{\circ}, \sigma=0.5\right.$, $\mathrm{k}_{\mathrm{b}}=9$.

\begin{tabular}{ccccc}
\hline O.V & B.V & BG.V & V & $\begin{array}{c}\text { Average } \\
\text { identification } \\
\text { rate }(\%)\end{array}$ \\
\hline 99.70 & 98.60 & 100 & 99.60 & 99.50 \\
\hline
\end{tabular}

Table 7. Variation of the scanning window size in function of the method used for an optimal classification result of SAR image

\begin{tabular}{cccc}
\hline Method & KLT-FB & GGM-FB & G-FB \\
\hline$k_{b}$ & 7 & 11 & 9 \\
\hline
\end{tabular}

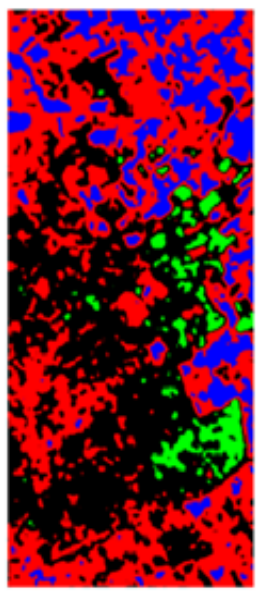

$\mathrm{a}$

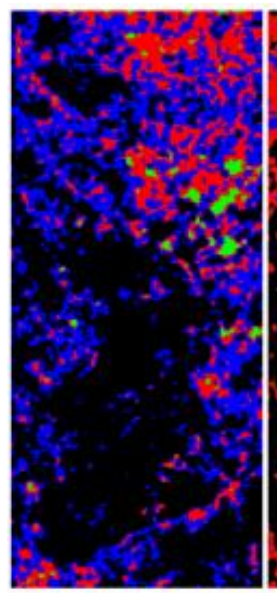

b

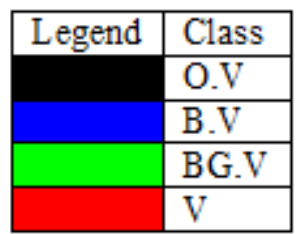

Fig.10. The best texture classification results of SAR image. (a)KLT-FB, (b) GGM-FB, (c) G-FB.

\section{DISCUSSIONS}

The three methods used for the development of the filter banks are quite complex in their implementation. GF method applied to the SAR image is estimated to be faster than the
GGM and KLT methods. In addition, it allows identifying all the textures of this image with classification rates up to $90 \%$ (see Fig.9).The complexity of the TKL method is due to the management of a very large number of filters by texture. However, the complexity of the GGM method is due the realization of the filters.

The textural identification results show that the average rates of the SAR textures identification are greater than $85 \%$ (see Tab.2, Tab.4, Tab.6). Also, it has been shown that the nature of the texture affects the performance of the different texture identification methods (see Fig.10). Indeed, the size of the scanning window $k_{b}$, representative of the texture, varies from an identification method to another (see Tab.7).

\section{CONCLUSION}

In this paper, different methods for the elaboration of the textural filter banks are presented. Only three methods among others are tested. The image used has been acquired with an airborne SAR sensor with high spatial resolution $(4.6 \mathrm{~m})$. Thus, the textures of this image are complex and present a mixture of primitives and therefore they are highly correlated. To reduce the degree of correlation, different orthogonal transforms were applied on our image by using the following methods: KLT, GF and the WT. We have shown that the built GF-FB permit us to reach an average textural identification rate of $99 \%$ of good classification, in the case of our SAR image textures. These results are more efficient than those obtained by the use of one of the transformations WT, FG or TKL methods without filtering operation. We have also mentioned that the effect of the size of the scanning window which is representative of the texture can gives the best classification results; however, the effect is variable from one method to another. This can be explained by the spatial transformation of the image.

\section{REFERENCES}

[1] Marrakchi Charfi O. "Elaboration de signatures des textures d'images de télédéction" Revue Française de photogrammétrie et de Télédétection, 2013, 11, (2), pp.337-350.

[2] Provent, P., Lemoine, J., Petit, E. : « Segmentation optimale d'images de texture par transformations adaptés multiples », Traitement du Signal, 1994, 11, (1), pp. 43-56.

[3] Devaux, J.C., Gouton, P., Truchetet, F.: « Karhunen-Loeve transform applied to region-based segmentation of color aerial images », Optical Engineering, 2001, 40, (7), pp. 1302-1308.

[4] Le Cadet, O. : « Méthodes d'ondelettes pour la segmentation d'images Application à l'imagerie médicale et au tatouage d'image ", Phd Thesis, Institut National Polytechnique de Grenoble, 2003.

[5] Laine, A., Fan, J.: « Frame representation for texture segmentation », IEEE Transactions on Image Processing, 1996, 5, (5), pp. 771-780.

[6] Mallat, S.: «Wavelet for a vision », Proceedings of the IEEE, 1996, 84 (4), pp. 604-614

[7] Chang, T., Kuo, C. C.: « Texture analysis and classification with Tree-Structured wavelet Transform », IEEE Transactions on Image Processing, 1993, 2, (4), pp. 429-441.

[8] Ben Amor, N., Ben Amara, N. : « Analyse texturale de l'écriture Arabe multifonte de Gabor aux Contourlet». Proc. 10ième Colloque International Francophone sur l'Écrit et le Document, Oct. 2008, France, pp.67-72.

[9] Weldon, TP., Higgins, WE.: «Algorithm for designing multiple Gabor filters for segmenting multi-textured images », Proc. IEEE International Conf. on Image Processing, Oct. 1998, Chicago, Illinois, USA, pp. 333-337.

[10] Yang, F. and Lishman, R.: « Land Cover Change Detection Using Gabor Filter Texture» Proc. 3rd International workshop on texture analysis and synthesis, Oct. 2003, Nice, France, pp. 113-118. 
[11] Bigün, J., Du Buf, J.H.: « N-folded symmetries by complex moments in Gabor space and their application to unsupervised texture segmentation », IEEE Transactions on Pattern Analysis and Machine Intelligence, 1994, 16, (1), pp. 80-87.

[12] Chen, J., Sato, Y., Tamura, S.: « Orientation Space Filtering for Multiple Line Segmentation», IEEE Transactions on Pattern Analysis and Machine Intelligence, 2000, 22, (5), pp. 417-429.

[13] Mbainaibeye, J., Marrakchi Charfi, O.: « Radar Image Texture Classification based on Gabor Filter Bank », International Journal of Electronics Communication and Computer Engineering, 2014, 5, (1), pp. 186-196.

[14] Marrakchi Charfi, O., Mbainaibeye, J.: « Performance study of the smart networks for remote sensing image textures identification», Word Scientific and Engineering Academy and Society Transactions on Signal Processing. 2016, 12, pp.179-191.

[15] Marrakchi Charfi, O., Mbainaibeye, J., Jebalia, W.: « Wavelet-Based Remote Sensing Heterogeneous Textures Signatures Using Generalized Gaussian Density Model », International Review on Computers and Software, 2012, 7, (2). pp. 538-545.

[16] Zand M., Doraisamy S., Abdul Halin A., Mas Rina M.: « Texture classification and discrimination for region-based image retrieval», Journal of Visual Communication and Image Representation, 2015, 26. pp. 305-316.

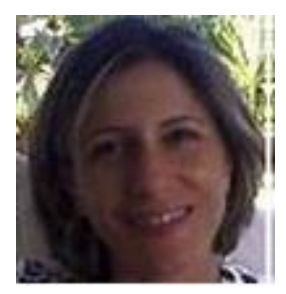

Dr. Olfa Marrakchi Charfi was born in Tunis, Tunisia, in 1968. She received the M.S. and $\mathrm{Ph} . \mathrm{D}$. degrees in electrical engineering, in Telecoms speciality, from the National School of Engineering of Tunis (ENIT), in 1995 and 1999, respectively. In 2000, she joined the National Institute of Applied Sciences and Technology of Tunis, where she is presently an Associate Assistant. During 1994-1999, she was with the Spatial Reference Systems and Remote Sensing Laboratory of ENIT. From 2003 to 2012, she was with Laboratory of Systems and Signal Processing of ENIT. Since 2015 she has joined the Natural Resources Management Laboratory of National Institute of Agronomy of Tunis (INAT), specialized in spatial detection, spatial analysis and modeling "GREEN-TEAM Laboratory", and she is interested in the characterization of arboreal surfaces and moisture soils using satellite data, in optics and in radar domains. Her research interests include image processing, learning machines, textures analysis, discrete wavelet transform (DWT) and applications of signal processing to remote sensing (RS). Currently, her research emphasis is on RS textures characterization and RS data classification, neural network and support vector machines algorithms.

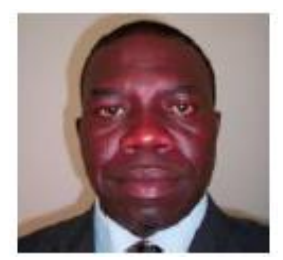

Dr. MBAINAIBEYE Jérôme have received the Master degree in Signal Processing and the PhD degree in Electrical engineering at the National High School of Engineers of Tunis, Tunisia, University of Tunis El Manar in October 1997 and July 2002 respectively. He was an Assistant Professor in the department of Computer Science at the Faculty of Sciences of Bizerte, Tunisia from 2002 to 2007. From May 20 to June 20, 2003, he was a scientific visitor at "ÉQUIPE SIGNAL et IMAGE of Ecole Nationale Supérieure d'Electronique, d'Informatique et de Radiocommunication de Bordeaux (E.N.S.E.I.R.B), University of Bordeaux I, France. In 2008 he has joined the Faculty of Applied and Exact Sciences in the University of N'djamena, Chad, as an Assistant Professor in Electrical Engineering. Since April 2012, he has joined the Polytechnic High Institute of Mongo (Chad) as Director General. In February $14^{\text {th }}, 2017$, he has joined the University of Doba (Chad) as Rector. Since July 2014, he is an Associate Professor of Electrical Engineer. $\mathrm{He}$ is member of Signal, Image and Pattern Recognition Laboratory of the National High School of Engineers, Tunis, Tunisia and an associated researcher in XLM Signal, Images and Communication laboratory department, University of Poitiers, France. He has published several papers in scientific journals and in international conference proceedings. He also has served as a reviewer of the IEEE International Conference on Sciences of Electronics, Technology of Information and Telecommunications SETIT'2007, the International Conference on Document Analysis and Recognition, ICDAR 2007 , and the IEEE $3^{\text {th }}$ International Conference on Information \& Communication Technologies: From Theory to Application, ICTTA' 2008. His research activities include
Digital Signal Processing, Image Processing, Image Analysis, Image and Video Compression, Communication Technologies, Wavelet Transform and its applications, Biometric systems and applications. 